\title{
MOBILE DEVICE OF VOIP VOICE TRAFFIC ANALYSIS FOR RED5 SERVICE
}

\author{
Jeanne Chen \\ National Taichung University of Science and Technology \\ No.129, Sec. 3, Sanmin Rd., North Dist., Taichung City 404, Taiwan \\ jeanne@nutc.edu.tw \\ Tung-Shou Chen \\ National Taichung University of Science and Technology \\ No.129, Sec. 3, Sanmin Rd., North Dist., Taichung City 404, Taiwan \\ tschen@nutc.edu.tw \\ Jhe-Wei Syu \\ National Taichung University of Science and Technology \\ No.129, Sec. 3, Sanmin Rd., North Dist., Taichung City 404, Taiwan \\ s18013103@nutc.edu.tw
}

\begin{abstract}
Due to the increase in popularity of mobile devices and mobile networks, the usage rate of VoIP has also increased. The flow consumption of types of VoIP has become very important from the limited mobile network data program. In recent years, the voice streaming server of Adobe Flash, Red5, has been increasingly used to realize the function of VoIP. Red5 is an open source media server and has good voice compression and video compression. However, it can only be executed on a PC computer. This is because Adobe Flash player has a hardware competence, such as microphone and camera lens. The flash phone is not supported or widely used on mobile devices. In this research, we propose a mobile device which can support Adobe AIR to obtain the competence of the hardware device and to use the Red5 library to communicate with the Red5 server. Experimental results showed that the proposed method allows mobile phones to call flash phones. Also, the flash phone bandwidth is about 4KBytes in comparison to the SIP phone, which makes it more suitable for use on mobile devices.
\end{abstract}

Keywords: VOIP, SIP, Flash Phone, TCP Mobile Network 


\section{INTRODUCTION}

Voice over Internet Protocol ${ }^{1}$ on the internet or other IP technology can be used in place of current telephone communication. This includes the ability to make calls through a variety of devices and network transmissions, where users benefit from not having to pay telecommunication fees. Costs may be saved for long distance and international calls. The increase in mobile internet and mobile device users also resulted in an increasing internet phone population where many people are using VoIP to cut down costs via network transmission costs.

In some countries, the mobile internet package is not an unlimited use of mobile network, so the packet size of VoIP has become very important. Therefore, when network transmission flow is smaller per second, talk time can be longer. The current most commonly used VoIP technology is Session Initiation Protocol (SIP) ${ }^{2,}{ }^{3}$. SIP phone can only be used on desktop computers, IP phones and mobile devices. Users need only go through the SIP server (such as Asterisk) for voices to talk with each other. The process requires only the cost of network traffic, for long distance and international calls. Transfer Protocol of SIP phone is Real-time Transport Protocol (RTP) ${ }^{3}$. It is based on UDP. Therefore, RTP does not ensure data transfer time or quality of service. Although RTP transmission speed can be very fast, it does not ensure whether a packet is served. This may result in packet loss. Currently, the SIP server Asterisk ${ }^{4}$ is used in VoIP where SIP server features are integrated for SIP communication exchange. In recent years, the new VoIP technique is to use Adobe Flash to create VoIP communications. Adobe Flash Player is developed by Adobe. And Adobe Flash phone is developed by the Infrared5 company to support flash phone media streaming server called $\operatorname{Red} 5^{5,6,7,8}$.Currently, the flash phone is made through a browser for phone calls where Real Time Messaging Protocol (RTMP) ${ }^{9,10,11}$ for voice or video streaming is used in comparison to SIP server RTP stream. The RTMP protocol is based on TCP, which has confirm-data-delivered characteristics so that transmission of voice will not result in packet loss due to discontinuous sound problems. However, RTMP is built on TCP with confirm-data-delivered characteristics, which require checks on messages causing higher packet traffic than UDP. Fortunately, the traffic cost is only $2 \mathrm{KBytes}^{8}$, which is suitable for applications on mobile devices. The current flash phone is confined only to use on desktop computers. The microphone and earpiece of current mobile devices are incompatible for use due to the outdated browser flash package for mobile phones. Our proposed method is to create a flash support mobile hardware kit, which will enable mobile devices to make calls on the flash phone. 
This paper is organized as follows: Section 2 explains related issues on flash phone and flash SIP phone. Section 3 introduces the proposed method. Section 4 describes the experimental results and Section 5 makes the final conclusions.

\section{RELATED WORK}

\subsection{Red5 Server and Flash Phone}

The flash phone client application is built on the Adobe flash action script 3.0 $0^{4,12}$. The Adobe Flash Player is required for use in flash phone. The Adobe Flash Player was developed for the purpose of making computers capable of using flash media for use of computer peripherals, such as the camera or microphone. Its common application is to use the browser to watch flash media. Currently, flash phone application clients use the flash phone. The Adobe Flash Player must be installed before flash phone can be used. This also requires the installation of flash phone media streaming server Red5 developed by the Infrared5 company for voice exchange in flash phone. Flash phone client voice transmission makes use of RTMP with Red5, mainly for the user to do call pairing. That is, when a user connects to Red5, the user ID will be recorded and when users dial the flash phone, Red5 will make connections based on the user ID. Figure 1 shows the Red5 server transmission of signal and voice exchange.

Flash phone internet traffic is a minimal $2 \mathrm{Kbytes}^{8}$. Therefore, flash for use on a mobile device is a good choice. But the flash phone client will need the Adobe Flash Player to work properly. The Adobe Flash Player is supported on mobile devices, but its hardware support is not ideal. The reason is the lack of permission from proprietary mobile devices, and security issues so the browser cannot be enabled on the phone hardware, such as a microphone and earpiece. Therefore, the mobile device with Adobe Flash Player application can only be used to watch flash media, but not for Flash-enabled hardware on the device.

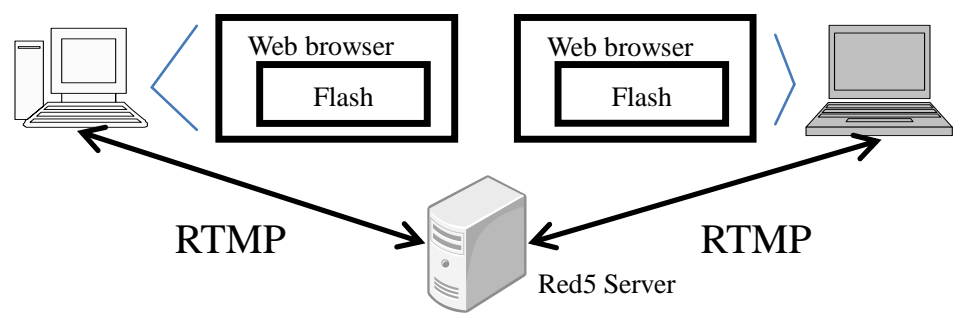

Figure 1. Flash phone structure ${ }^{8}$ 


\subsection{Flash Phone Call SIP Phone}

Currently, SIP is used to save costs on VoIP. However, there are problems with communication between flash phones and SIP phones. A bridge module would be required. The Infrared5 developed Red5 server also provides SIP phone calls, which could be used as a bridge interface for the flash phone to communicate with SIP phones. However, the application core for the flash phone client is built on Flash Action Script 3.0 architecture, which requires Adobe Flash Player. In order to communicate with the SIP phone, the SIP communication interface will have to be added to the flash phone. Flash phone SIP interfaces can be divided into Red5 server and Red5 SIP application.

First, the user receives and sends sounds by Red5 server transfer. Before Red5 server sends the microphone sound to the SIP phone, the first to arrive is the RTMP User Class module. This module is primarily used for RTMP streaming to deliver voice data and also for Red5 SIP application to receive or transfer signaling. When the RTMP User Class module sends voice to SIP phone, this will cause two sides with incompatible encoding. Therefore, voice cannot be directly sent to the SIP phone, but must be through the ASAO turn u-law module encoding and then sent to SIP User Class module. Finally, the SIP User Class module will be the voice sent to the SIP phone. The SIP User Class module is mainly used to receive voice from SIP phone or send voices and signals. When the SIP User class module receives SIP phone signals or voice calls, signals would be sent to the Red5 SIP application and voice callback to RTMP User Class module. The RTMP User Class module, on receiving the voice, will be compressed and transmitted to the Red5 server to be sent to the user's client end, where sound is played. In the second part, Red5 SIP application is mainly used to send or receive call signals. The signal content is dialed, accepted and listened to the call status. Red5 SIP application work is received or transmitted through signal transfer to the SIP User Class module or RTMP User Class module and client. It is the bridge of Red5 phone and SIP phone communication. Flash SIP phone interface process is as shown in Figure 2 where the client interface is built in two parts ${ }^{8}$. The first is to send and receive signals, and the other is to send and receive voice. RTMP User Class module and SIP User Class module are transceivers for signal and voice processing. Although flash phone to call the SIP phone can even have calls to support SIP on a mobile device, this method is limited to use on the desktop computer. The reason is the same problem as mentioned in section 2.1. This is because Red5 SIP phone application only provides the browser version, and Adobe Flash Player must be installed. The general mobile device browser support in the Adobe Flash Player and for the hardware is 
far from ideal due to proprietary and security issues in mobile devices where the browser cannot be enabled for phone hardware, such as a microphone and earpiece. Our proposed method provides a solution as described in section 3.

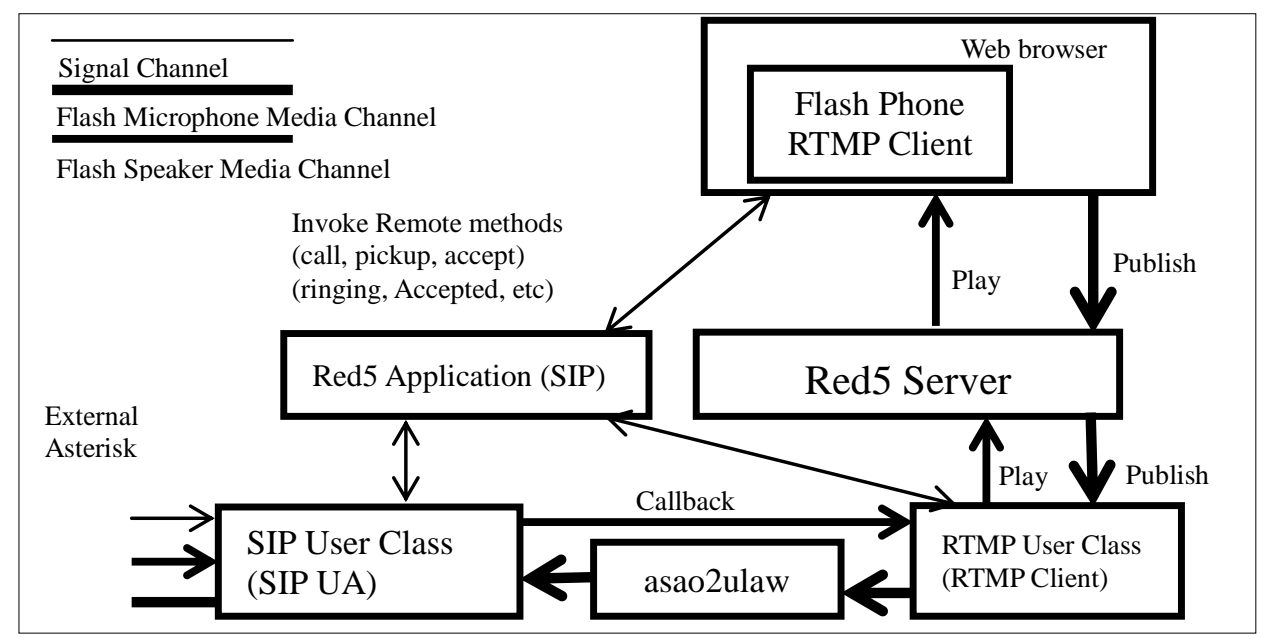

Figure 2. Flash SIP phone structure ${ }^{8}$

\section{THE PROPOSED METHOD}

In order to realize a mobile device making flash phone calls, we proposed a method based on Adobe AIR $^{12}$ to support flash in mobile devices, and with Flex development to combine the Red5 library to achieve support for mobile device flash phone applications. In this paper, the proposed method is divided into two parts.

The first part is to change structure of flash phone applications on the client end. Flash phone application is based on browser on desktop computers and also requires Adobe Flash Player. This architecture is not supported on mobile devices. Therefore, in this paper, the original flash phone end client application architecture is modified and the browser application is changed to mobile device application as shown in Figure 3. The original flash phone application browser version of the end client is converted into mobile applications to achieve the mobile flash phone application. The flash phone features mobile device hardware that can be supported in this implementation. The second part is to realize a flash phone mobile application_based on Adobe AIR development. The original flash phone application is built on Flash Action Script 3.0 architecture and flash phone mobile application architecture is the same. The only difference is that the software runs on a desktop computer using Adobe Flash Player and 
the mobile device uses Adobe AIR. However, the flash phone mobile application is just a program. Therefore, it does not have to rely on the browser to enable flash phone, where the flash phone application can make use of Adobe AIR to run its applications. Flash phone mobile application development environment is to use Flash Builder ${ }^{12}$ software development application and using Flex mobile project development with two libraries to achieve flash phone mobile application. The first library is the Red5 library. This library is the client end with Red5 server communication. The source is the original Red5 phone built-in Red5 library and can be found in Red5 Server developed by Infrared5.

Finally, the Adobe Native Extension (ANE) ${ }^{12}$ library used is Flash Action Script 3.0. The ANE library is Flex with mobile device source code communication tools. In this method, the main control hardware mobile device, such as the headset, will make use of ANE for headset permission for sound output from speaker. The improved structure is as shown in Figure 4. After completing ANE development for client end flash phone mobile application, no change is necessary for the Red5 server. The original problem in the flash phone was the hardware problem in the client end, which has nothing to do with the Red5 Server. After completing the Flash Phone Mobile Application and to enable microphone and headset, the hardware will be able to connect to the Red5 server for use in voice communication.

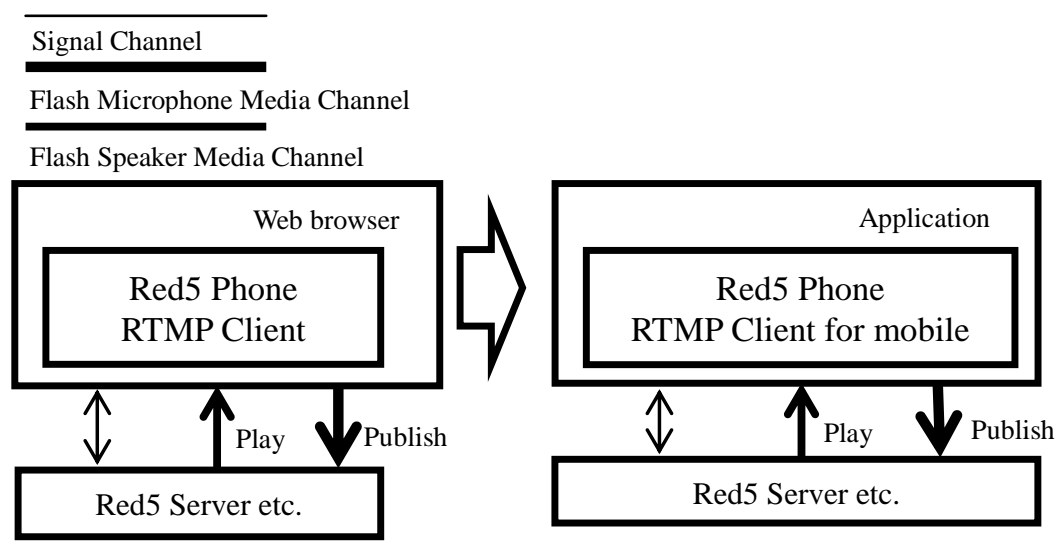

Figure 3. Flash phone browser version converted into flash phone mobile application 


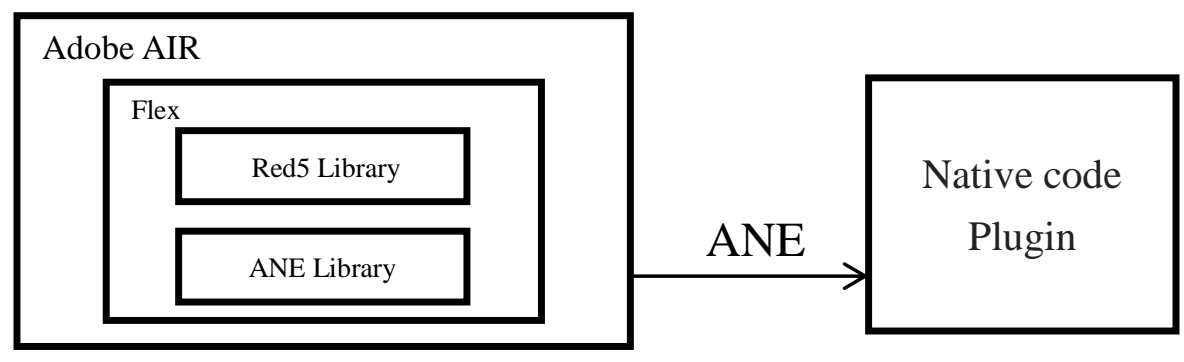

Figure 4. Flash phone mobile application structure

\section{EXPERIMENTAL RESULTS}

For the experimental tests, we proposed the server environment using Linux installed with Asterisk and Red5. Five combination tests were performed using two mobile devices. The aim is to compare the upload and download flow between Asterisk (SIP phone) and Red5 (Flash phone) and the combination of both. Each combination takes one hour. Table 1 shows the results of comparison for the upload and download flow for each combination. The audio sampling frequencies for the phones are in KHz. In test 1 (SIP phone to SIP phone at $8 \mathrm{KHz}$ vs. $8 \mathrm{KHz}$ ) comparison to test 3 (Flash phone to Flash phone of the same frequency) showed that flash phone resulted in higher voice quality. Also, the flash phone showed smaller packets than the SIP phone with a maximum difference of more than double. On the other hand, in test 4 (Flash phone to Flash phone at $8 \mathrm{KHz}$ vs. $22 \mathrm{KHz}$ ), the higher frequency resulted in higher voice quality. Assume a user with the $3 \mathrm{G}$ network is limited to 1GByte (upload plus download) using SIP phone to SIP phone for about 772 minutes of talk time, and flash phone to flash phone for 1,781 minutes of talk time. The talk time difference of both would be more than double. This study is helpful for users with limited internet bandwidth.

\section{CONCLUSION}

This paper presents a VoIP-based mobile device built on Red5 server applications. Adobe AIR and Flex are used to develop a flash phone client end to support hardware for phone communications. Experimental results showed that flash phone traffic flow is smaller than SIP phones on mobile devices. For users with limited mobile internet traffic, this is a useful feature for voice communication. Communication time can be extended without extra costs. Users are also able to adjust the quality of microphone sound quality without dropping communications. 
Table 1. Internet traffic experimental result for SIP phone and flash phone

\begin{tabular}{llcc}
\hline \multicolumn{1}{c}{ Experimental Tests } & $\begin{array}{c}\text { Upload } \\
\text { Kbytes/sec }\end{array}$ & $\begin{array}{c}\text { Download } \\
\text { Kbytes/sec }\end{array}$ \\
\hline 1 & SIP phone to SIP phone & $10.78-11.13$ & $9.97-10.47$ \\
2 & $\begin{array}{l}\text { Flash phone to SIP phone } \\
\text { Flash phone to Flash phone (8KHz vs. }\end{array}$ & $4.58-5.15$ & $4.03-4.41$ \\
3 & $\begin{array}{l}\text { 8KHz) } \\
\text { Flash phone to Flash phone (8KHz vs. }\end{array}$ & $4.3-4.78$ & $4.03-4.3$ \\
4 & $\begin{array}{l}\text { 22KHz) } \\
\text { Flash phone to Flash phone (22KHz vs. }\end{array}$ & $7.38-8.01$ \\
5 & $\begin{array}{l}\text { 22KHz) } \\
\text { 22-8.35 }\end{array}$ & $6.75-7.55$ \\
\hline
\end{tabular}

\section{REFERENCES}

[1] K. Nisara, A. Amphawana, S. Hassana, and N.I. Sarkarb, A comprehensive survey on scheduler for VoIP over WLAN. Journal of Network and Computer Applications, 36(2), p933-948, 2013. http://dx.doi.org/10.1016/j.jnca.2012.07.019.

[2] J.S. Leu, W.H. Lin, W.B. Hsieh, and C.C Lo, Design and implementation of a VoIP broadcasting service over embedded systems in a heterogeneous network environment. The Scientific World Journal, 2014(1), p1-10, 2014. http://dx.doi.org/10.1155/2014/917060.

[3] B. Campbell, J. Rosenberg, H. Schulzrinne, C. Huitema, and D. Gurle, Session initiation protocol(SIP) extension for instant messaging. Retrieved on March 20, 2015, from http:// http://tools.ietf.org/pdf/rfc3428.pdf.

[4] Asterisk, Asterisk*. Retrieved on November 20, 2013, from http://www.asterisk.org/.

[5] X.F. Gong, and D.M. Zhang, Research on web server based on red5, tomcat and apache. Advanced Materials Research, 282-283, p721-725, 2011. http:// 10.4028/www.scientific.net/AMR.282-283.721.

[6] M. Osamnia, A.J. Berena, S. Chunwijitra, H. Okada, and H. Ueno, A cloud-based multi-functional e-meeting system by flash-based multimedia technology for higher education on the WebELS system. Lecture Notes in Computer Science, 8294, p1-12, 2013. http://dx.doi.org/10.1007/978-3-319-03731-8_1.

[7] BigBlueButton Source Web bigbluebutton, Bigbluebutton open source web conferencing. Retrieved on March 20, 2015, from http://code.google.com/p/bigbluebutton/.

[8] S. Gong, P. Gregoire, and D. Rossi, Red5-Reference Documentation. Retrieved on March 20, 2015, from 
http://red5.googlecode.com/svn-history/r3744/doc/trunk/reference/pdf/ red5-reference-0.9-en.pdf (2012).

[9] H.J. Kim, and S.G. Choi, QoE assessment model for multimedia streaming services using QoS parameters. Multimedia Tools and Applications, $\quad 72(3), \quad$ p2163-2175, 2014. http://dx.doi.org/10.1007/s11042.

[10] P. Zhao, J. Li, J. Xi, and X. Gou, A mobile real-time video system using RTMP. In G.S. Tomar, T.N. Sharma and T.N. Sharma (Eds.), Proceedings of 2012 Fourth International Conference on Computational Intelligence and Communication Networks (CICN) (p61-64). Mathura: IEEE Press, 2012. http://dx.doi.org/10.1109/CICN.2012.18.

[11] H. Parmar, and M. Thornburgh, Adobe's real-time messaging protocol. Retrieved on March 20, 2015, from http://www.adobe.com/content/dam/Adobe/en/devnet/rtmp/pdf/rtmp_s pecification_1.0.pdf (2012).

[12] Adobe Air introduction, Adobe flash runtimes/Adobe AIR. Retrieved on March 20, 2015, from http://www.adobe.com/products/air.html. 
